\title{
Slow-Releasing Tranilast in Polytetrafluoroethylene/ Polylactide-co-glycolide Laminate Delays Adjustment after Strabismus Surgery in Rabbit Model
}

\author{
Min Joung Lee, ${ }^{1,2}$ Su-Eon Jin, ${ }^{2,3}$ Chong-Kook Kim, ${ }^{3}$ Ho-Kyung Choung, ${ }^{4}$ Jin Wook Jeoung, ${ }^{1}$ \\ Hwa Jung Kim, ${ }^{5}$ Gheeyoung Choe, ${ }^{6}$ and Jeong-Min Hwang ${ }^{7}$
}

Purpose. To determine the usefulness of slow-releasing tranilast in polytetrafluoroethylene/polylactide-co-glycolide (PTFE/PLGA) laminate for delayed adjustable strabismus surgery.

Methods. A prospective, masked-observer, controlled study was performed in 25 rabbits. Fifty rabbit eyes were divided randomly into three groups. After a recession of the superior rectus muscle (SRM), a PTFE/PLGA laminate containing tranilast, PTFE alone, or balanced salt solution was applied beneath and over the SRM in the PTFE/PLGA-tranilast group (group P-T), the PTFE group (group P), and the control group, respectively. Delayed adjustment was performed by a masked observer once on each SRM 3 or 5 weeks after surgery. Adjustability, adjustment lengths, forces required, and adhesion degrees were evaluated.

REsults. In the control group, adjustment was impossible in any eye 3 or 5 weeks after surgery. In group $P$, adjustment was possible in 5 of 8 eyes 3 weeks after surgery and in 5 of 10 eyes 5 weeks after surgery. In group P-T, adjustment was possible in 8 of 10 eyes 3 and 5 weeks after surgery. On comparing adjustability, a significant difference was observed between group P-T and the control group 3 and 5 weeks after surgery ( $P=0.006, P=0.006$, respectively). A significant difference was observed between group P-T and the control group $(P=$ 0.016) in terms of adhesion between SRMs and conjunctivae 5 weeks after surgery.

Conclusions. Slow-releasing tranilast in PTFE/PLGA was found to reduce adhesion and allowed delayed adjustment in most eyes for up to 5 weeks after surgery. (Invest Ophthalmol Vis Sci. 2007;48:699-704) DOI:10.1167/iovs.06-0775

From the ${ }^{1}$ Department of Ophthalmology, Seoul National University College of Medicine, Seoul National University Hospital, Seoul, Korea; ${ }^{3}$ Laboratory of Excellency for Drug and Gene Delivery, College of Pharmacy, Seoul National University, Seoul, Korea; ${ }^{4}$ Department of Ophthalmology, Seoul National University College of Medicine, Seoul National University Boramae Hospital, Seoul, Korea; ${ }^{5}$ Pharmacoepidemiology Division, Clinical Trial Center, Seoul National University Hospital, Seoul, Korea; and Departments of ${ }^{6}$ Pathology and ${ }^{7}$ Ophthalmology, College of Medicine Seoul National University, Seoul National University Bundang Hospital, Seongnam, Korea.

${ }^{2}$ These authors contributed equally to the work presented here and should therefore be regarded as equivalent authors.

Submitted for publication July 7, 2006; revised August 2 and September 8, 2006; accepted December 13, 2006.

Disclosure: M.J. Lee, None; S.-E. Jin, None; C.-K. Kim, None; H.-K. Choung, None; J.W. Jeoung, None; H.J. Kim, None; G. Choe, None; J.-M. Hwang, None

The publication costs of this article were defrayed in part by page charge payment. This article must therefore be marked "advertisement" in accordance with 18 U.S.C. $\$ 1734$ solely to indicate this fact.

Corresponding author: Jeong-Min Hwang, Department of Ophthalmology, College of Medicine Seoul National University, Seoul National University Bundang Hospital, 300 Gumi-dong, Bundang-gu, Seongnam, Gyeonggi 463-707, Korea; hjm@snu.ac.kr.
A djustable suture strabismus surgery has become an effective method of adjusting binocular alignment during the immediate postoperative period. ${ }^{1,2}$ The ability to correct undercorrection or overcorrection and to lower the reoperation rate is the main advantage of adjustable suture strabismus surgery. However, binocular alignment may drift over time, even after eyes are placed in a suitable position by adjustable strabismus surgery. ${ }^{3}$ Therefore, delayed adjustment avoiding the effects of anesthesia and muscle injury after initial surgery may produce better postoperative results. ${ }^{4,5}$ However, the postoperative healing process causes adhesions, which inhibit such delayed adjustment.

Efforts have been made to facilitate delayed adjustment by implanting physical barriers such as silicone, ${ }^{5,6}$ viscoelastic material, ${ }^{7}$ absorbable adhesion barrier (Interceed TC7; Johnson \& Johnson Medical Inc., Arlington, TX), ${ }^{8}$ polyglactin 910 mesh, ${ }^{8}$ polytetrafluoroethylene (PTFE, W.L. Gore \& Associates, Inc., Flagstaff, AZ), ${ }^{9}$ or bioresorbable film (SurgiWrap; MacroPore Biosurgery, San Diego, CA), ${ }^{10}$ antiproliferative agents such as mitomycin $\mathrm{C}^{11}$ or combinations of these various physical barriers and antiproliferative agents. ${ }^{12-14}$ Adjustment can be delayed by using silicone for up to 11 days in humans ${ }^{5}$ and for up to 8 weeks in rabbits. ${ }^{6}$ However, silicone can cause discomfort because of its thickness and rigidity, and it may trigger infection, extrusion, or granuloma formation. Polytetrafluoroethylene (PTFE) barriers have also been shown to allow delayed adjustment for up to 4 weeks and to have some advantages over other physical barriers. ${ }^{9,13}$ All barriers, however, induce foreign body reactions and require frequent instillations of anti-inflammatory agents.

Polylactide-co-glycolide (PLGA) copolymer, a type of biodegradable polymer, has been extensively studied because it is degraded by the simple hydrolysis of ester bonds into lactic and glycolic acids, which are removed by normal metabolic pathways. ${ }^{15}$ PLGA is one of the few biodegradable polymers approved for human clinical use by the Food and Drug Administration. ${ }^{16}$ Moreover, it has been used for site-specific controlled drug release over extended periods in the eyes. ${ }^{17}$ Thus, this offers the possibility of a drug delivery system that allows the slow release of an anti-inflammatory or anti-adhesive drug at a sustained therapeutic concentration over several days or weeks. Recently, PTFE/PLGA containing dexamethasone was reported to allow delayed adjustment for up to 5 weeks in rabbit eyes. ${ }^{18}$ However, this steroid agent may have adverse effects such as intraocular pressure elevation and cataract formation, which can be clinically significant given that the drug is released over several weeks.

Tranilast, $N$-[3,4-dimethoxycinnamoyl] anthranilic acid, was developed as an anti-allergy drug and has been found to prevent the inflammation and collagen synthesis derived from keloid and hypertropic scarring, perhaps by inhibiting TGF- $\beta 1$ secretion. ${ }^{19}$ Tranilast eyedrops are widely used to treat allergic conjunctivitis, and they have negligible toxic effects. ${ }^{20}$ Thus, we considered designing a system capable of continually releasing tranilast to prevent postoperative adhesions after stra- 
bismus surgery. In this experimental study, we evaluated the effectiveness of PTFE/PLGA film containing tranilast to facilitate delayed adjustment after strabismus surgery in a rabbit model.

\section{Materials ANd Methods}

All experiments were conducted in accordance with the ARVO Statement for the Use of Animals in Ophthalmic and Vision Research. The protocol was approved by the Institutional Animal Care and Use Committee of Seoul National University Hospital.

Twenty-five New Zealand White rabbits, each weighing 2.0 to 3.0 $\mathrm{kg}$, underwent 5-mm recession of the superior rectus muscles (SRMs) with the use of double-armed, nonabsorbable 5-0 polyester (5-0 Ethibond; Ethicon, Somerville, NJ) sutures and were subsequently adjusted 3 and 5 weeks after surgery. PTFE/PLGA containing sustained-release tranilast was used as a physical barrier in group P-T, whereas PTFE not containing tranilast was used in group P. In the control group (group C), no physical barrier was used. Fifty SRMs were divided among three experimental groups as follows: group $\mathrm{C}(10$ muscles; 5 for examination at 3 weeks and 5 at 5 weeks), group P (20 muscles; 10 for examination at 3 weeks and 10 at 5 weeks), and group P-T (20 muscles; 10 for examination at 3 weeks and 10 at 5 weeks). Two eyes of each rabbit were randomly assigned to three groups to minimize inter-rabbit variation.

\section{Procedures}

General anesthesia was induced by administering 30 to $45 \mathrm{mg} / \mathrm{kg}$ ketamine hydrochloride and 5 to $10 \mathrm{mg} / \mathrm{kg}$ xylazine hydrochloride intramuscularly, and topical anesthesia was achieved using proparacaine hydrochloride. Muscle recession procedures with or without a physical barrier were performed as previously described. ${ }^{18}$

Polyvinylpyrrolidone-iodine was applied to the eyelids for preoperative surgical antisepsis. Limbal peritomy was performed from 10 o'clock to 2 o'clock. The SRM was isolated on a Jameson hook, and intermuscular connections were dissected. The superior oblique tendon was disinserted and allowed to retract from the surgical field. The SRM was then placed on a double-armed 5-0 polyester (5-0 Ethibond; Ethicon) suture close to the insertion and disinserted from the globe. In group P-T (20 SRMs), a separate sheet $(1 \times 2 \mathrm{~cm})$ of PTFE/PLGA containing tranilast was placed between the sclera and the SRM and folded to separate the conjunctiva and the SRM. An identical process was used in the PTFE without tranilast group (group P; 20 SRMs), but no barrier was inserted in the control group (10 SRMs). Finally, the SRM was recessed $5 \mathrm{~mm}$ and reattached to the original insertion using a hang-back suture technique. A bucket-handle suture was made for future traction. The edges of the conjunctival peritomy were approximated with interrupted 8-0 polyglactin sutures. At the end of each procedure, ofloxacin eye ointment was applied topically, and $4 \mathrm{mg}$ gentamicin was injected into a thigh muscle.

\section{Delayed Adjustment}

In a masked random fashion, the SRMs were adjusted under anesthesia 3 or 5 weeks after surgery in all three groups. The delayed adjustment procedure was performed as follows. The previous conjunctival incision site was opened with the tip of a curved needle holder or scissors. After the SRM had been fully exposed, a PTFE or a PTFE/PLGA membrane was removed before adjustment. A dial tension gauge (DT-50; Teclock Inc., Okaya, Japan) was used to precisely control the amount of force applied. The noose suture attached to the SRM was hooked with the bar of a dial tension gauge. The SRM was then moved anteriorly as much as possible. This adjustable distance was measured with a Castroviejo caliper, and the force required was measured with the gauge.

\section{Preparation of Polymeric Film Fabrication}

Polylactide-co-glycolide 50:50 (PLGA; average molecular weight, 40,000 -75,000; Sigma, St. Louis, MO) containing tranilast (Choongwae Pharma Corp., Seoul, Korea) was cast onto PTFE film with a solvent casting technique. Briefly, tranilast (144 mg) and PLGA (1000 mg) were dissolved in $10 \mathrm{~mL}$ acetone (Fisher Scientific, Suwanee, GA) with stirring for 30 minutes at room temperature. Blank and drug-containing PLGA solutions were cast onto glass plates and onto PTFE film-coated glass plates. Cast polymer films were dried in a refrigerator for 48 hours to allow the acetone to evaporate slowly and then were dried for 24 hours at room temperature. PLGA films and PTFE films coated with PLGA were peeled from the glass plates and cut into pieces measuring $1 \mathrm{~cm} \times 1 \mathrm{~cm}$. Drug loadings on film weighed approximately 1.0 $\mathrm{mg} / \mathrm{cm}^{2}$.

\section{Determination of Tranilast Loading PLGA}

Polymeric films were dissolved in $2 \mathrm{~mL}$ acetonitrile and vortexed for 1 minute. The polymer solutions obtained were then filtered through a $0.45-\mu \mathrm{m}$ membrane filter to remove precipitated material; Acetonitrile $(4.5 \mathrm{~mL})$ was then added to $0.5 \mathrm{~mL}$ of filtrate and vortexed for 30 seconds. One hundred microliters of this solution was diluted to 900 $\mu \mathrm{L}$ with mobile phase, and a $10-\mu \mathrm{L}$ aliquot was directly injected into a high-performance liquid chromatograph. Tranilast loadings were then determined as previously described. ${ }^{21}$

The high-performance liquid chromatography (HPLC) system comprised a separation module (2690; Waters, Milford, MA) with pump, autosampler, UV detector (2487; Waters), and $\mathrm{C}_{18}$ reversed-phase column (Capcellpak MGII, $5 \mu \mathrm{m}, 250 \mathrm{~mm} \times 1.5 \mathrm{~mm}$; Shiseido, Tokyo, Japan). Data were analyzed (MassLynx software; Micromass, Manchester, UK). The mobile phase consisted of $40 \%$ (vol/vol) acetonitrile and $60 \%$ (vol/vol) $10 \mathrm{mM}$ phosphate buffer ( $\mathrm{pH} \mathrm{3.5).} \mathrm{The} \mathrm{volume} \mathrm{injected}$ was $10 \mu \mathrm{L}$, and the flow rate used was $0.10 \mathrm{~mL} / \mathrm{min}$. Under these conditions, linear calibration curves of tranilast were obtained in the concentration range 0.5 to $8 \mu \mathrm{g} / \mathrm{mL}\left(r^{2}>0.99\right)$.

\section{In Vitro Release of Tranilast}

Tranilast release from the polymer laminate was studied in phosphatebuffered saline (PBS). A polymer film sample $(1 \mathrm{~cm} \times 1 \mathrm{~cm})$ was placed in $23 \mathrm{~mL}$ PBS ( $\mathrm{pH} 7.4$ ) containing $0.1 \%$ sodium azide as a preservative, and magnetically stirred in a circulating water bath $37^{\circ} \mathrm{C}$. At predetermined times $(0,0.17,0.25,0.33,0.42,1,2,3,5,6,7,10,15,17,21,27$, and 30 days), $400-\mu \mathrm{L}$ aliquots of the medium were collected, replaced with an equal volume of fresh PBS, and filtered through a $0.45-\mu \mathrm{m}$ membrane filter. Ten microliters of this solution was directly injected to the HPLC for tranilast quantification. In vitro release experiments were repeated three times to obtain a release profile of tranilast.

\section{Evaluation of Adhesions}

At the time of adjustment, adhesions between muscle, PTFE or PTFE/ PLGA membrane, sclera, and conjunctiva were evaluated and recorded. Adhesions were classified as SRM/C (superior rectus muscle/ conjunctiva) or as SRM/S (superior rectus muscle/sclera) when they were, respectively, located above or below an SRM. Adhesion severities were scored from 0 to 4 according to criteria described in previous report, ${ }^{18}$ as follows: $0=$ no adhesion; $1=$ filmy adhesion easily separable with blunt dissection; $2=$ mild to moderate adhesion with freely dissectible plane; 3 = moderate to dense adhesion with difficult dissection; and $4=$ nondissectible plane. Animals were killed after delayed suture adjustment by administering a $10-\mathrm{mL}$ intravenous injection of sodium pentothal.

\section{Postmortem Histologic Examination}

Histopathologic studies were performed to evaluate inflammation and fibrosis between sclera, SRM, and conjunctiva. Tissues in each of three groups were examined macroscopically and microscopically using hematoxylin and eosin staining and Masson trichrome staining. 


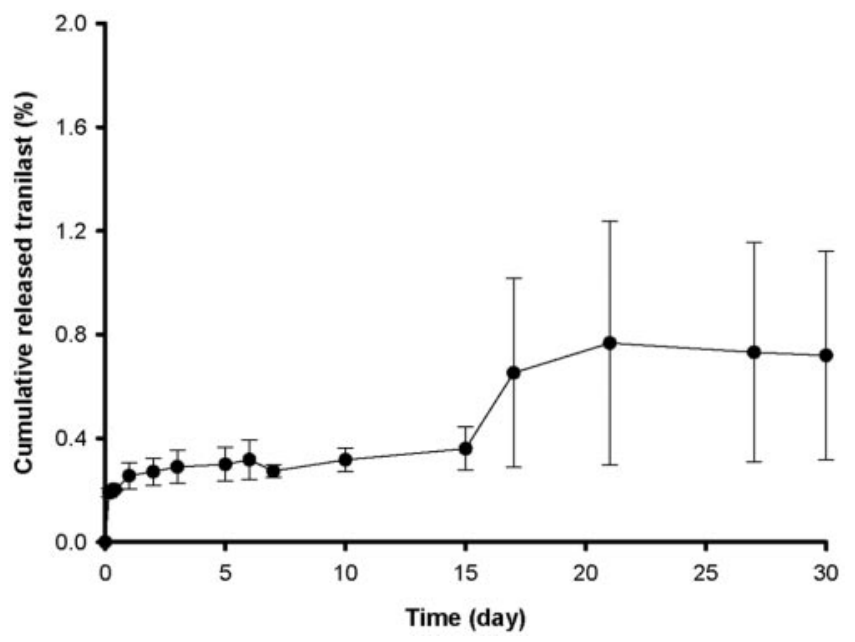

FIGURE 1. In vitro cumulative release profile of tranilast from tranilastpoly (DL-lactide-co-glycolide) 50:50 (PLGA)- coated polytetrafluoroethylene film. The release profile of tranilast from PTFE/PLGA film was studied in PBS at $\mathrm{pH} 7.4$ and $37^{\circ} \mathrm{C}(n=3)$. The total amount of tranilast released into $\mathrm{PBS}$ was expressed as a percentage of the total amount of tranilast loaded into the PTFE/PLGA film.

\section{Statistical Analyses}

Statistical analyses were performed with the Fisher exact test to identify difference among three groups with respect to adjustability. Advancement distances and forces needed to advance SRMs were analyzed with the Mann-Whitney $U$ test. For statistical analysis, adhesion severities were divided into low (score 0, 1, 2) or high (score 3, 4) grade and compared with the Fisher exact test. Statistical significance was accepted for $P$ values $\leq 0.05$. When statistical significance was identified among three groups, post hoc pairwise comparisons adjusted by Bonferroni method were carried out.

\section{Results}

During the follow-up period, before adjustment, 2 eyes were lost because of the death of a single rabbit. The two eyes were incidentally included in group $\mathrm{P}$.

\section{In Vitro Release of Tranilast}

The release of tranilast from PTFE/PLGA film was found to occur in a triphasic manner, with an initial release burst followed by a low-release phase and a high-release phase (Fig. 1). The initial release of tranilast was probably the result of the release of tranilast localized at the surface, and release during the second and third phases probably resulted from a combi- nation of drug diffusion through the PLGA film and hydrolytic degradation of PLGA.

\section{Adjustability}

In the control group, adjustment was impossible in any eye 3 and 5 weeks after surgery. In group $P$, adjustment was possible in 5 of 8 eyes 3 weeks after surgery and in 5 of 10 eyes at 5 weeks after surgery. In group P-T, adjustment was possible in 8 of 10 eyes at 3 weeks and 5 weeks after surgery (Table 1).

On comparing adjustability 3 and 5 weeks after surgery, a significant difference was found among three groups $(P=$ $0.013, P=0.015$, respectively). Subsequent pairwise comparisons showed that the difference between group P-T and the control group was significant $(P=0.006, P=0.006$, respectively; Bonferroni corrected). No significant difference was observed between groups $\mathrm{P}$ and P-T or between group $\mathrm{P}$ and the control group 3 weeks $(P=0.608, P=0.075$, respectively) or 5 weeks after surgery $(P=0.349, P=0.101$, respectively $)$.

\section{Adjustment Amount and Force Required}

In group $\mathrm{P}$, average adjustment (advancement) amount and force were $2.10 \mathrm{~mm}$ and $54.00 \mathrm{~g} 3$ weeks after surgery and 2.35 $\mathrm{mm}$ and $50.80 \mathrm{~g} 5$ weeks after surgery. In group P-T, the average adjustment amount and force were $2.31 \mathrm{~mm}$ and $34.12 \mathrm{~g} 3$ weeks and $2.43 \mathrm{~mm}$ and $42.75 \mathrm{~g} 5$ weeks (Table 1 ). On comparing adjust amount, no difference was found between groups $\mathrm{P}$ and $\mathrm{P}-\mathrm{T}$ at 3 or 5 weeks after surgery $(P=0.943, P=$ 0.724 , respectively). For adjust forces, a significant difference was found between groups $\mathrm{P}$ and P-T at 3 weeks after surgery $(P=0.030)$ but not at 5 weeks after surgery $(P=0.524)$.

\section{Degree of Adhesion between SRM and Conjunctiva}

A significant difference was observed among three groups with respect to the degree of adhesion between SRMs and conjunctivae 3 or 5 weeks after surgery $(P=0.002, P=0.023$, respectively). Pairwise comparisons revealed significant differences between groups $\mathrm{P}$ and P-T $(P=0.001$; Bonferroni corrected) 3 weeks after surgery and between group P-T and the control group $(P=0.016$; Bonferroni corrected $) 5$ weeks after surgery. No significant difference was observed between the control group and group $\mathrm{P}(P=0.102)$ or between the control group and group P-T $(P=0.333) 3$ weeks after surgery, and no significant difference was observed between group $\mathrm{P}$ and the control group $(P=0.113)$ or between group $\mathrm{P}$ and group $\mathrm{P}-\mathrm{T}$ $(P=0.582) 5$ weeks after surgery (Fig. 2).

\section{Degree of Adhesion between SRM and Sclera}

No significant difference was observed among three groups in terms of degree of adhesion between SRMs and sclerae 3

TABLE 1. Numbers of Adjustable Eyes and Adjustment Tractional Forces and Distances

\begin{tabular}{cccccc}
\hline Group & $\begin{array}{c}\text { Time } \\
\text { (wk) }\end{array}$ & $\begin{array}{c}\text { Adjustment } \\
\text { Possible } \\
\text { (no. eyes) }\end{array}$ & $\begin{array}{c}\text { Adjustment } \\
\text { Not possible } \\
\text { (no. eyes) }\end{array}$ & $\begin{array}{c}\text { Force } \\
(\mathbf{g})\end{array}$ & $\begin{array}{c}\text { Length } \\
\text { (mm) }\end{array}$ \\
\hline C & 3 & 0 & 5 & $*$ & $*$ \\
& 5 & 0 & 5 & $*$ & $*$ \\
P & 3 & 5 & 3 & $54.00 \pm 15.03$ & $2.10 \pm 0.55$ \\
& 5 & 5 & 5 & $50.80 \pm 21.94$ & $2.35 \pm 0.70$ \\
P-T & 3 & 8 & 2 & $34.12 \pm 9.92$ & $2.31 \pm 1.09$ \\
& 5 & 8 & 2 & $42.75 \pm 10.74$ & $2.43 \pm 0.86$ \\
\hline
\end{tabular}

Values for force and length are mean \pm SD. C, control; P, polytetrafluoroethylene; P-T, polytetrafluoroethylene/polylactide-co-glycolide + tranilast.

* Impossible to move the muscle because of adhesion. 
(A)

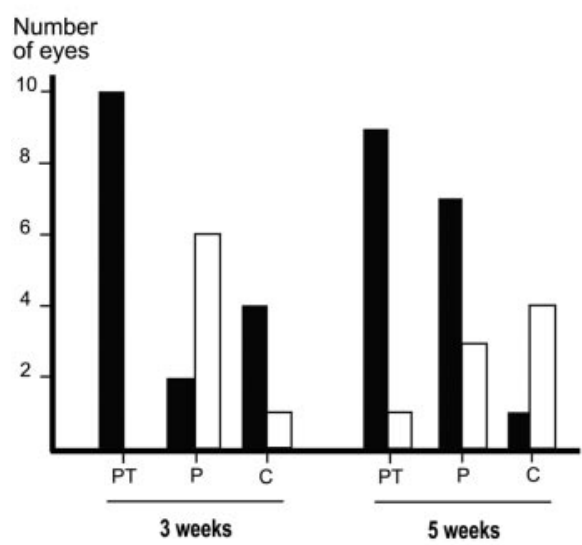

(B)

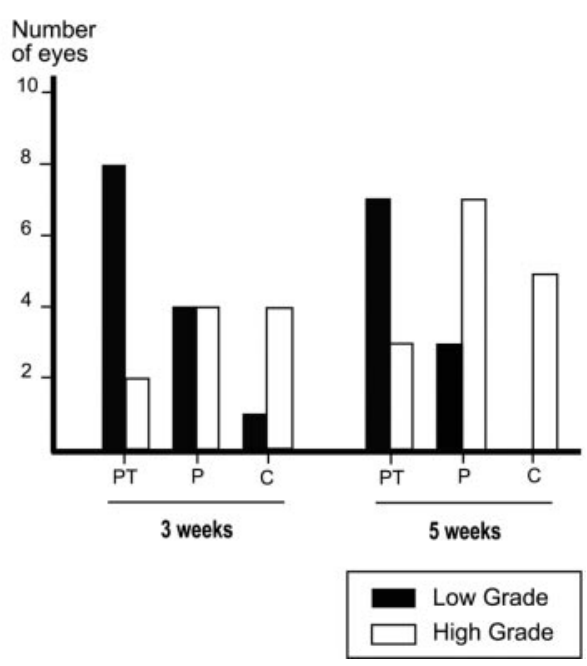

Figure 2. Comparison of the degree of adhesion between groups. (A) Adhesion degree between superior rectus muscle and conjunctiva. The degree of adhesion was low in most eyes in group P-T at 3 or 5 weeks after surgery. A significant difference was observed between group $\mathrm{P}-\mathrm{T}$ and group $\mathrm{P}(P=0.001) 3$ weeks after surgery and between group P-T and the control group $(P=0.016) 5$ weeks after surgery. (B) Adhesion degree between superior rectus muscle and sclera. No significant difference was observed among three groups 3 weeks after surgery. At 5 weeks after surgery, the degree of adhesion was high in most eyes in group $\mathrm{P}$ and the control group. C, control; $\mathrm{P}$, polytetrafluoroethylene; P-T, polytetrafluoroethylene/polylactide-co-glycolide + tranilast. weeks after surgery $(P=0.099)$. A significant difference was observed 5 weeks after surgery $(P=0.033)$, but the post hoc pairwise comparisons did not show any significant differences between three groups. No significant difference was observed between group $\mathrm{P}$ and the control group $(P=0.505)$ or between groups $\mathrm{P}$ and P-T $(P=0.177) 5$ weeks after surgery. A considerable difference was observed between group P-T and the control group at this time but did not reach significance ( $P=0.026$; Bonferroni corrected; Fig. 2).

\section{Histologic Examination}

Histologic examination using with hematoxylin and eosin staining showed some degrees of inflammation with fibrosis around SRMs in the control group and in group P 3 weeks after surgery, and these were reduced 5 weeks after surgery. Mild inflammation and fibrosis were observed around SRMs in group P-T. Masson trichrome staining also revealed that fibrosis was least prominent in group $\mathrm{P}-\mathrm{T}$ compared to that in group $\mathrm{P}$ or

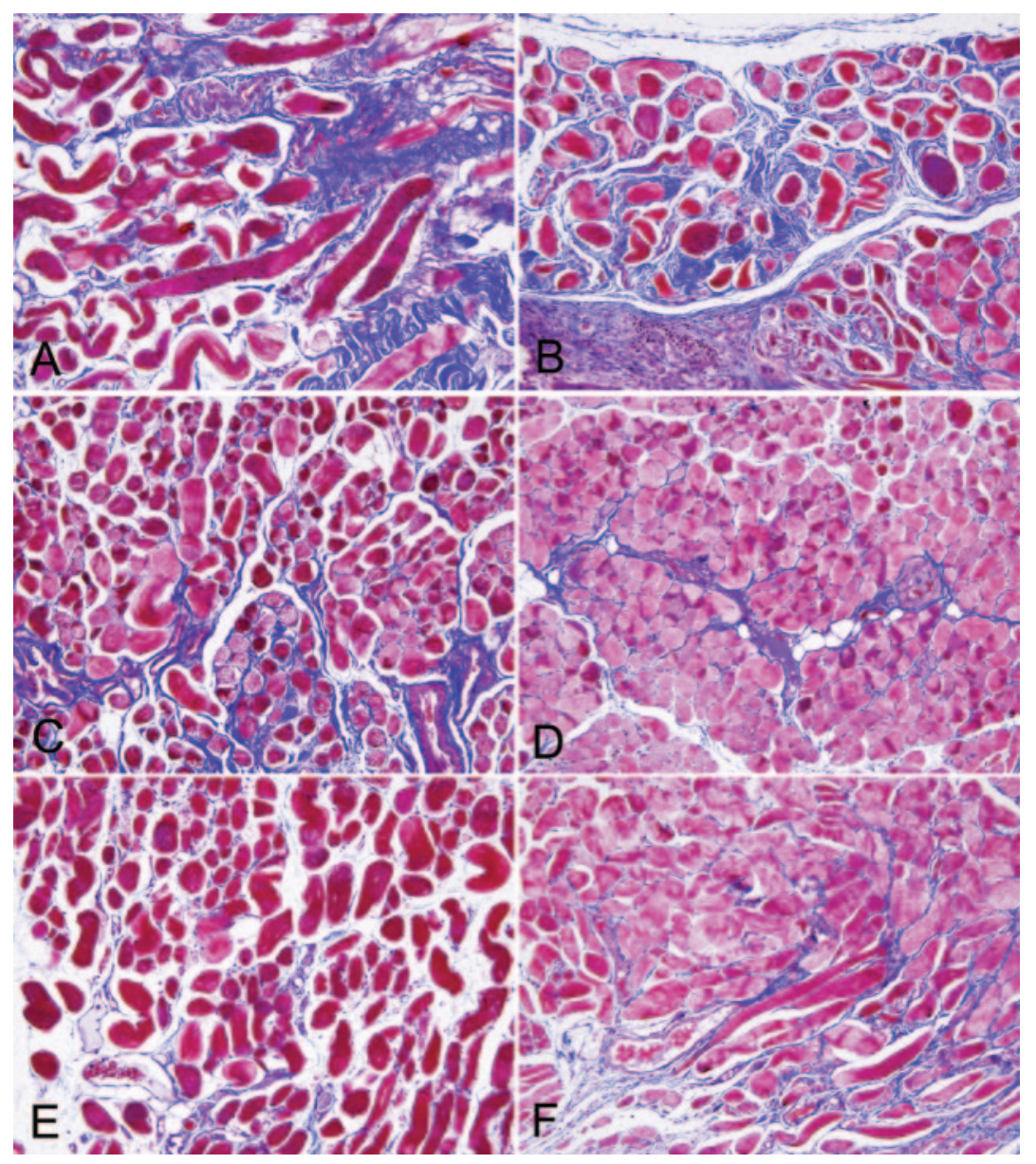

Figure 3. Light microscopic findings (Masson trichrome; original magnification, $\times 200$ ) of the superior rectus muscle 3 weeks $(\mathbf{A}, \mathbf{C}, \mathbf{E})$ and 5 weeks $(\mathbf{B}, \mathbf{D}, \mathbf{F})$ after surgery. (A, B) Control group. $(\mathbf{C}, \mathbf{D})$ Group $P$. (E, F) Group P-T. 
the control group (Fig. 3). No specific toxic reaction was observed in group $\mathrm{P}$ or group P-T.

\section{Discussion}

PTFE has been used as pericardial and as abdominal patch graft material for several years and as an alloplastic xenograft for the treatment of eyelid and socket diseases or in glaucoma surgery. ${ }^{22-29}$ PTFE is chemically and biologically inert, nonantigenic, and resistant to infection, and it is easily cut, molded, and sutured. ${ }^{22}$ It is available as an expanded porous $1.0-\mathrm{mm}-$ thick sheet or as a $0.1-\mathrm{mm}$ nonporous thin surgical membrane. Nonporous 0.1-mm PTFE is soft and flexible and can be applied to complex shapes without difficulty. Moreover, because of its thinness, it is well tolerated by patients and generally does not cause discomfort. Given these advantages, we considered PTFE the most suitable nonabsorbable material choice for delaying adjustment.

The ideal drug-release device for our purpose would fulfill the following criteria. ${ }^{27}$ First, it should provide a sustained and relatively uniform therapeutic drug release in a reliable and predictable manner over a required period. Second, the device should be easily implantable or injectable but remain stable and not migrate within the eye. Third, the device should have a long shelf life, and it should be easily handled and sterilize. Moreover, because the conjunctival incision must be reopened to adjust sutures, unlike other intraocular devices, it does not have to be biodegradable. None of the sustained delivery systems introduced to date fulfill these criteria.

In the present study, a $0.1-\mathrm{mm}$-thick PTFE film coated with PLGA was used. PLGA film is difficult to manipulate because it tends to roll up and stick to itself, ${ }^{14}$ but PTFE coated with PLGA is easier to handle.

Our previous study suggested that PTFE alone might allow adjustment to be delayed for up to 4 weeks after surgery in $40 \%$ of rabbit eyes 9 and that the combined use of PTFE and 5-fluorouracil, or the addition of viscoelastic solution (Viscoat; Alcon Laboratories, Fort Worth, TX) could allow adjustment to be delayed for up to 4 weeks after surgery in $80 \%$ of rabbit eyes. $^{13}$

In this study, we evaluated adjustability 3 weeks and 5 weeks after surgery based on a release profile of tranilast, which showed sustained release up to 4 weeks. Only group P-T was found to be significantly more adjustable than the control group 3 and 5 weeks after surgery. Moreover, only group P-T showed significantly less adhesion between SRMs and conjunctivae 3 and 5 weeks after surgery. In addition, the present study produced favorable results without the need for frequent topical steroid instillation. These results demonstrate that PTFE/ PLGA, which allowed sustained tranilast release, effectively prevented adhesion development and improved adjustability after strabismus surgery in our rabbit model.

Tranilast is known to inhibit the release of histamine, leukotriene, interleukin, and TGF- $\beta$ and to suppress the initial stages of the wound-healing processes such as vascular leakage, fibrin deposition, and migration of monocytes and fibroblasts. ${ }^{28-30}$ In this study, the histopathologic findings of group P-T showed minimal inflammation and fibrosis 3 and 5 weeks after surgery even though we did not evaluate the degree of inflammation or fibrosis quantitatively compared with other groups.

Tranilast has also been evaluated in some ocular diseases. Chikara et al. ${ }^{31}$ reported the use of topical tranilast after filtering surgery reduced intraocular pressure and increased the size of the bleb. In addition, an in vitro study showed tranilast inhibited rabbit tenon capsule fibroblast and collagen synthesis. ${ }^{32}$ Tranilast is also thought to reduce corneal haze after photorefractive keratectomy. ${ }^{33}$
Delaying adjustment allows the surgeon to determine better where a patient's motility will stabilize. In humans, Hwang (Hwang JM, unpublished data, March 1998) showed that delayed adjustable strabismus surgery is possible with the use of a thin polytetrafluoroethylene plate, and Shokida et al. ${ }^{34}$ found that delayed adjustment surgery using a silicone sheet produced better results in exotropia patients who underwent reoperation than in patients who underwent immediate adjustment. The results of the present study suggest that tranilastreleasing PTFE/PLGA could be useful in delaying adjustment up to 5 weeks.

In conclusion, we describe a new drug and a new drugdelivery system based on the extraocular sustained release of tranilast from a PTFE/PLGA film. This system was found to provide significantly better adjustability in delayed suture strabismus surgery in rabbit eyes for up to 5 weeks after surgery. Moreover, this study demonstrates for the first time the effects of tranilast on delayed suture strabismus surgery.

\section{References}

1. Jampolsky A. Strabismus reoperation techniques. Trans Am Acad Ophthalmol Otolaryngol. 1975;79:704-717.

2. Rosenbaum AL, Metz HS, Carlson M, et al. Adjustable rectus muscle recession surgery: a follow-up study. Arch Ophthalmol. 1977;95: 817-820.

3. Cassin B, Serianni N, Romano P. The change in ocular alignment between the first day and six weeks following eye muscle surgery. Am Orthoptic J. 1986;36:99-107.

4. Howard CW, Smith AG. Use of adjustable sutures: a helpful modification. Ann Ophthalmol. 1986;18:70-73.

5. Shokida MF. Use of silicone sheet for delayed adjustable strabismus surgery. Ophthalmic Surg. 1993;24:486- 488.

6. Nemet P, Stolovitch C. Delayed reattachment of extraocular muscle. Binocul Vis Strabismus. 1989;4:23-26.

7. Hwang JM, Chang BL. Use of Viscoat for delayed postoperative adjustable suture strabismus surgery in rabbits. Binocul Vis Strabismus. 1996;11:137-142.

8. Hwang JM, Chang BL. Use of physical barriers for delayed adjustable strabismus surgery; the effect of Interceed and polyglactin 910 mesh. Br J Ophthalmol. 1996;80:759-762.

9. Hwang JM, Chang BL. Delayed reattachment of extraocular muscles in rabbits using thin polytetrafluoroethylene. Ophthalmic Surg Lasers. 1997;28:59-64.

10. Choung HK, Hwang JM. The use of Surgi Wrap ${ }^{\mathrm{TM}}$ in delayed adjustable strabismus surgery. Am J Ophthalmol. 2005;140:433436

11. Oh SO, Chang BL, Lee J. Effect of mitomycin C on delayed adjustment in experimental strabismus surgery. Korean J Ophthalmol. 1995;9:51-58.

12. Hwang JM, Chang BL. Combined effect of Interceed and 5-fluorouracil on delayed adjustable strabismus surgery. Br J Ophthalmol. 1999;83:788-791.

13. Hwang JM, Chang BL. Combined effect of polytetrafluoroethylene and 5-fluorouracil on delayed adjustable strabismus surgery. $J \mathbf{P e}$ diatr Ophthalmol Strabismus. 2000;37:163-167.

14. Kim JH, Jeong SY, Jung MH, Hwang JM. Use of polyurethane with sustained release tranilast in delayed adjustable strabismus surgery. Br J Ophthalmol. 2004;88:1450-1454.

15. Gilding DK. Biodegradable polymers. In: Williams DF, ed. Biocompatibility of Clinical Implant Materials. Vol. 2. Boca Raton, FL: CRC Press; 1981:209-232.

16. Vert M, Christel P, Chabot F, Leray J. Bioresorbable plastic materials for bone surgery. In: Hastings GW, Ducheyne P, eds. Macromolecular Biomaterials. Boca Raton, FL: CRC Press; 1984:119142.

17. Giordano GG, Arroyo MH, Refojo MF. Sustained delivery of retinoic acid from microspheres of biodegradable polymer in PVR. Invest Ophthalmol Vis Sci. 1993;34:2743-2751.

18. Jee JP, Choung HK, Kim CK, Hwang JM. Polytetrafluoroethylene/ polylactide-co-glycolide laminate containing dexamethasone al- 
lows delayed adjustable strabismus surgery in a rabbit model. Invest Opbthalmol Vis Sci. 2006;47:2485-2490.

19. Suzawa H, Kikuchi S, Ichikawa K. Inhibitory effect of an antiallergic drug, tranilast, on the production of cytokines. Pbarmacokinetics. 1992;43:409-414.

20. Nagata R, Arimura S, Samejima H, et al. Frequent eye irritation study of a short term and subacute toxicity of tranilast (N-5') ophthalmic solution in rabbits. Clin Rep. 1992;26:3463-3469.

21. Lamiable D, Vistelle R, Millart $H$, et al. High-performance liquid chromatographic determination of dexamethasone in human plasma. J Chromatogr. 1986;378:486-491.

22. Hanel KC, McCabe C, Abbott WM, Fallon J, Megerman JL. Current PTFE grafts: a biochemical, scanning electron, and light microscopic evaluation. Ann Surg. 1982;195:456-463.

23. Slader JC, Maxwell FM. Experience with 30 polytetrafluoroethylene grafts. Am J Surg. 1981;141:546-548.

24. Karesh JW, Putterman AM. Reconstruction of the partially contracted ocular socket or fornix. Arch Opbthalmol. 1988;106:552556.

25. Steinkogler FJ, Kuchar A, Huber E, Arocker-Mettinger E. Gore-Tex soft-tissue patch frontalis suspension technique in congenital ptosis and in blepharophimosis-ptosis syndrome. Plast Reconstr Surg. 1993;92:1057-1060.

26. Hwang JM, Kee C. The effect of surface area expansion with pericardial membrane (Preclude ${ }^{\circledR}$ ) in Ahmed glaucoma valve implant surgery. J Glaucoma. 2004;13:335-339.
27. Ferruti P, Tanzi MC. New polymeric and oligomeric matrices as drug carriers. Crit Rev Ther Drug Carrier Syst. 1986;2:175-244.

28. Komatsu H, Kojima M, Tsutsumi N, et al. Mechanism of inhibitory action of tranilast on release of slow reacting substance on anaphylaxis (SRS-A) in vitro: effect of tranilast on the release of arachidonic acid and its metabolites. Jpn J Pbarmacol. 1988;46: 53-60.

29. Suzawa H, Kikuchi S, Ichikawa K, et al. Inhibitory action of tranilast, an anti-allergic drug, on the release of cytokines and PGE2 from human monocytes-macrophages. Jpn J Pharmacol. 1992;60: 85-90.

30. Yamada H, Tajima S, Nishikawa $T$, et al. Tranilast, a selective inhibitor of collagen synthesis in human skin fibroblasts. $J$ Biochem. 1994;116:892-897.

31. Chikara E, Dong J, Ochiai H, Hamada S. Effects of tranilast on filtering blebs: a pilot study. J Glaucoma. 2002;11:127-133.

32. Oshima T, Kurosaka D, Kato $\mathrm{K}$, et al. Tranilast inhibits cell proliferation and collagen synthesis by rabbit corneal and Tenon's capsule fibroblasts. Curr Eye Res. 2000;20:283-286.

33. Okamoto S, Sakai T, Iwaki Y, et al. Effects of tranilast on cultured rabbit corneal keratocytes and corneal haze after photorefractive keratectomy. Jpn J Ophthalmol. 1999;43:355-362.

34. Shokida F, Melek N, Dominguez D, Ciancia A. Delayed adjustment in strabismus surgery with silicone sheet versus early adjustment. Am Ortbopt J. 1997;47:152-156. 